\title{
Especial y marginal: hacia una problematización de la intervención estatal en la salud de los veteranos de Malvinas (1984-2000)
}

\author{
Special and marginal: towards a problematization of \\ the state intervention regarding the health of Malvinas \\ veterans (1984-2000)
}

${ }^{1}$ Doctor en Ciencias Sociales. Becario postdoctoral, Instituto de Investigaciones Geohistóricas, Consejo Nacional de Investigaciones Científicas y Técnicas (IIGHICONICET), Resistencia, Chaco, Argentina. $\triangle$ (iD
RESUMEN El objetivo de este artículo es dar cuenta de las modalidades en que fue instituida la salud del veterano argentino de la guerra de Malvinas como un problema de intervención estatal entre 1984 y 2000. Para ello nos centramos en el concepto de problematización como un modo de análisis de las prácticas y el pensamiento político. El texto consta de tres apartados: en el primero se presentan las bases metodológicas para el análisis de las políticas públicas, mientras que el segundo y el tercero están destinados al análisis de una serie de leyes, proyectos de ley, decretos, informes, etc., producidos por diferentes esferas estatales en torno al veterano de guerra y su situación sanitaria. La hipótesis de lectura que proponemos es que el veterano o excombatiente de Malvinas fue problematizado como un segmento poblacional marginal, aunque la estabilización de un tratamiento específico tardó más de quince años en materializarse.

PALABRAS CLAVES Salud de los Veteranos; Islas Malvinas; Políticas de Salud; Gobierno Estatal; Argentina.

\begin{abstract}
The aim of this paper is to account for the modalities in which Malvinas veterans' health was constituted as a problem requiring state intervention between 1984 and 2000. In order to do so, we have focused on the concept of problematization as a way to analyze practices and political thought. The text consists of three sections: the first one presents the methodological basis of the analysis of public policies, whereas the second and third ones intend to analyze a series of laws, bills, decrees, reports and other documents produced by different state spheres about war veterans and their health situation. The hypothesis that we propose is that the Malvinas veterans were problematized as a marginal segment of the population, although the stabilization of a specific treatment took more than fifteen years to materialize.
\end{abstract}

KEY WORDS Veterans Health; Malvinas Islands; Health Policy; State Government; Argentina. 


\section{INTRODUCCIÓN}

La guerra de Malvinas (abril-junio de 1982), no solo significó una derrota militar para Argentina en manos de Gran Bretaña y una de las principales trabas para lograr la soberanía sobre las islas, también fue un acontecimiento político, social y cultural de gran magnitud que afectó en diversas formas a la sociedad postconflicto. En estas líneas nos ocuparemos de lo sucedido en la posguerra con un sujeto emergente: el excombatiente o veterano de guerra. Nos interesa remarcar fundamentalmente los modos de problematización política acaecidos sobre este sujeto a partir de las formas en que fueron presentados sus problemas, especialmente, la conexión que se hizo entre la necesidad de reintegración social y los efectos que la guerra les produjo. Para ello, nos ocuparemos de rastrear las formas en que, en democracia, fue configurada una situación especial como plafón para el gobierno de los veteranos de guerra. Es decir, nuestro objetivo es mostrar las maneras en que fue definido este sujeto novedoso (la noción de veterano de guerra o excombatiente emergió en el campo político argentino tras la guerra y no antes, es decir, que la legislación nacional no preveía qué hacer con sus ciudadanos en armas tras un posible escenario de guerra) desde diversos espacios institucionales estatales, a partir de presentarlo como un problema de intervención política-estatal en materia de salud. La hipótesis de lectura que proponemos es que el veterano o excombatiente de Malvinas fue problematizado como un segmento poblacional marginal, aunque la estabilización de un tratamiento específico tardó más de quince años en materializarse.

En este sentido, el artículo se propone no solo contribuir al estudio histórico del tratamiento de salud de los veteranos, sino también en aportar a la reflexión sobre la ligazón entre guerra y posguerra pues entendemos que la definición de los veteranos como un segmento de la población anticipó los modos en que otras prácticas políticas segmentaron otros grupos, cuestión característica de las políticas de salud en la Argentina de la década de 1990 y posterior. El desarrollo de este texto forma parte de la tesis doctoral denominada Problematización y gobierno de los veteranos de la guerra de Malvinas. Estado nacional, intervención política y gubernamentalidad (1982-2017) y retoma algunas preguntas que hemos establecido en otros trabajos ${ }^{(1)}$. La escritura se organiza en tres apartados y una conclusión, desde los cuales rearmamos la red desplegada en torno a la salud de los veteranos que se centró en dos modos de pensar el problema. Una parte de las prácticas hizo énfasis en la necesidad de una convocatoria general y totalizadora a partir de las juntas de reconocimiento médico; la otra, centró sus esfuerzos en la calidad de cobertura de salud y los grados de dependencia de esta respecto a otras circunstancias (discapacidad, asistencia). El período abarcado inicia en el primer año postdictadura (septiembre de 1984) hasta los inicios del siglo XXI. En ese transcurso, identificamos dos momentos: un primer momento, que inició con una propuesta de acción estatal a gran escala que permitió imaginar diagnósticos en serie mediante las juntas de reconocimiento médico que posibilitarían reparaciones puntuales a casos individuales; $y$, un segundo momento, que se ligó al problema entre la ineficiencia de las juntas y la necesidad de atención personal y familiar de los ex soldados. En el hiato entre la especificidad y la asistencia fue produciéndose el camino a una atención especial que tuvo en el PAMI (entidad que brinda servicios médicos y sociales a personas mayores de 70 años sin jubilación y a ex combatientes de Malvinas) su principal objetivo y en el corrimiento del diagnóstico previo su novedad. No obstante, el tratamiento específico tardó más de 20 años en estabilizarse.

\section{EL ESTUDIO HISTÓRICO DE LO POLÍTICO A TRAVÉS DE LAS PROBLEMATIZACIONES}

Apenas finalizado el conflicto bélico en junio de 1982, miembros de las diversas Fuerzas Armadas y la propia Junta Militar (que 
gobernaron de facto el país entre 1976 y 1983 con un saldo de 30 mil desaparecidos y cientos de muertos y exiliados) empezaron a señalar que su propio futuro dependería, entre otras cosas, de la forma en que la sociedad inscribiera la guerra en su imaginario. En ese sentido, una de las primeras prerrogativas fue establecer un apoyo a los veteranos de guerra, con el objetivo de contribuir con su recuperación moral, psíquica y física para superar las consecuencias de la guerra. El grupo de seres humanos partícipe en las batallas se convirtió, en la imaginación militar, en uno de los sostenes para mejorar la alicaída imagen pública de la Junta. Posteriormente, y como señaló Rosana Guber ${ }^{(2)}$, las instituciones militares intentaron incorporar a los conscriptos a su sistema de salud (como modo de control), pero rápidamente el sistema se mostró colapsado e ineficiente. Al respecto, Andrea Rodríguez señala que:

...en cada unidad castrense se creó la Oficina de Malvinas para hacer el seguimiento del personal afectado, asignar beneficios y evaluar méritos o faltas de conducción militar [...]. El sistema de salud incluía no solo a los hospitales castrenses, sino también algunos institutos privados que colaboraron por propia voluntad [...]. Si bien la estructura hospitalaria fue desbordada, en algunos casos, los ex-soldados se negaban a acercarse a las instituciones sanitarias dependientes de las FFAA. ${ }^{(3)}$

Más allá del éxito o fracaso de estas iniciativas, nos interesa movernos en las maneras en que son presentadas, es decir, en los modos en que una serie de sujetos que se presentan como portavoces del Estado postulan, describen, dan forma a una realidad a partir de la explicitación de sus deseos de modificarla. Inscribimos nuestro enfoque en la perspectiva foucaultiana de análisis del gobierno ${ }^{(4)}$, y denominamos problematización a la puesta en discurso de ese deseo, a partir de la propuesta analítica de Carol Bacchi. Para la autora la práctica política, para poder desplegarse e intervenir, presenta a la realidad como un problema. Es decir, las diversas soluciones que establecen una ley, un proyecto, un decreto o un discurso de autoridad, no van por fuera del proceso a resolver, sino que "son producidas como problemas particulares junto a la política y las propuestas de políticas" (they are produced as problems of particular kinds within policies and policy proposals). A nivel de análisis, la mirada debe posicionarse en las soluciones postuladas en un determinado programa de gobierno y preguntarse "si se sugiere que esta forma de cambio o intervención es requerida, ¿cuál es el problema representado en cuestión?" (if the suggestion is that this form of change or intervention is required, what is the problem represented to be? $)^{(5)}$.

Este punto de vista se aleja de pensar la intervención política como reactiva para pasar a ver el pensamiento político como productivo y creativo, enfocándose en la representación del problema, los supuestos de los que parte, los silencios que produce, los efectos buscados, los espacios donde se difunden estas soluciones. La propuesta articula las nociones de problematización y gubernamentalidad, a partir de establecer que lo que se gobierna son los problemas que hacen a lo real pensable e intervenible ${ }^{(6)}$. La noción de problematización gubernamental nos permite inferir que toda acción de gobierno está vinculada a una problematización y a la delimitación de un perímetro mediante el cual se promueven modos de acción, de guía, de conducción que solucionen problemas a sujetos, a quienes se definen mediante objetos que se muestran más reales que otros, y en espacios que se presentan como mejores que otros. El enfoque posibilita el estudio histórico de una serie de problemas a partir de vincularlos por los grados de cercanía que postulan (la noción de problematización tiene algunos puntos de encuentro con enfoques sobre problemas socialmente construidos $^{(5)}$, pero discute la predominancia que estos le dan a la disputa de actores o por el contrario al énfasis en condiciones estructurantes foráneas a las prácticas) $)^{(7)}$.

Asimismo, y a nivel metodológico, nos valemos de la noción de red concebida por 
Latour $^{(8)}$ para dar cuenta de los ensambles de textos prácticos ${ }^{(9)}$ anclados y ritualizados como estatales, que presentan al veterano como un problema a intervenir.

\section{EL NACIMIENTO DE UNA SITUACIÓN ESPECIAL: LOS VETERANOS Y SUS SECUELAS}

En nuestro país el sistema de salud presenta un esquema tripartito (un sistema solidario ligado a la actividad laboral conocido como obras sociales, uno público y uno privado conocido como prepaga) con objetivos de cobertura universal pero que en la práctica lejos está de ello. Si bien todos los ciudadanos están protegidos

...un grupo de la población tiene cober-
turas múltiples y de diferente calidad por
la superposición de la oferta pública y
la cobertura obligatoria de la seguridad
social. Por otro lado, la organización
descentralizada y las características terri-
toriales del país han determinado un
acceso diferente para cada grupo pobla-
cional. ${ }^{(10)}$

Entre las décadas de 1970 y 1980, el país asistió a un proceso de deterioro del sistema público que, con el tiempo, se focalizó en atender a quienes no podían acceder a las otras dos partes del esquema tripartito, imbricándose con las políticas asistenciales.

No obstante, las primeras discusiones sobre la tecnología sanitaria que se activaría respecto a los ex conscriptos no ponían en duda la capacidad del sistema público ni militar. La mayor invención de la década de 1980 respecto de las políticas hacia los veteranos -nos referimos a la Ley 23109 de Beneficios a Ex Combatientes, que establecía reconocimientos en salud, trabajo, vivienda y educación- planteó como objeto de mayor importancia el reconocimiento médico, esto es, un diagnóstico de salud dirigido a la masa de jóvenes veteranos no militares, y su reparación inmediata. Recordemos que Argentina entró en guerra bajo un sistema de conscriptos (una mixtura entre soldados profesionales y civiles que cumplían el servicio militar obligatorio). Según datos oficiales, casi el 50\% de los soldados o personal de apoyo movilizados por la Junta Militar no eran pro18fesionales: de los 10.289 pertenecientes al Ejército, 7.540 fueron soldados conscriptos y 76 eran civiles que cumplían funciones de apoyatura; de los 10.596 de la Armada, 3.540 eran conscriptos y 469 civiles; y de los 2.314 movilizados por la Fuerza Aérea, 459 era conscriptos y 48 civiles. Esto significaba que una parte importante de los protagonistas de la guerra no contaría, una vez finalizada, con protecciones estatales de ningún tipo pues quedaban afuera de la cobertura militar.

La Ley 23109 partió de dos proyectos presentados casi en simultáneo. El de los senadores Francisco Villada y Deolindo Bittel, por un lado, y el del senador Oraldo Britos, los tres pertenecientes al Partido Justicialista (Partido Justicialista). En el primero se argumentó que los ex soldados "soportan de por vida las secuelas, las lesiones físicas y psíquicas"(11) y planteó como obligación de Estado la normalización de sus condiciones de vida. Asimismo, este proyecto se ligó con el de Britos quien propugnó la realización de una convocatoria nacional y la conformación de tribunales especiales "integrados por un profesional médico por cada una de las fuerzas, un profesional médico del Ministerio de Salud y un profesional médico designado por el Ministerio de Trabajo y Seguridad Social"(12) para evaluar el estado de salud y poder decidir el tratamiento y la reparación pecuniaria a quienes se había desatendido en sus necesidades vitales para "superar las secuelas propias de su participación en el conflicto". No es difícil imaginar la ligazón entre la secuela y la desprotección, pues la primera sería la marca de los desprotegidos.

En el dictamen de comisiones, el armado tecnológico de salud se diferenció del proyectado por Britos, pues planteó un grado de mayor racionalidad. La propuesta final que entró en debate fue emplazar a estos sujetos con secuelas en las "Juntas de Reconocimiento Médico que funcionan en las 
delegaciones Sanitarias Federales del Ministerio de Salud y Acción Social [...] exista o no dictamen anterior de junta médica de las respectivas fuerzas"(13). En definitiva, la definición y autenticidad de la marca quedaría en manos civiles $y$, posteriormente, al determinarse las "secuelas psicofísicas derivadas de su participación en el conflicto, la fuerza en la que este prestó servicio deberá hacerse cargo de la atención médica y de todos los gastos que demande el completo restablecimiento del interesado".

El debate en Cámara Alta se llevó a cabo rápidamente tras el dictamen, en el que el senador Antonio Berhongaray por la Unión Cívica Radical sintetizó las metas de la ley respecto a la salud: "Establecimos una convocatoria obligatoria para que todos sean sometidos nuevamente a revisión médica porque, de acuerdo con algunas denuncias realizadas, las revisiones no resultaron muy exactas o precisas" ${ }^{\prime \prime 14)}$.

La propuesta pasó a la Cámara de Diputados donde se aprobó casi sin debates, y la ley fue promulgada y publicada en octubre. Sin embargo, el instrumento no tuvo decreto de reglamentación hasta 1988 y la convocatoria obligatoria nunca se llevó a cabo. Al respecto, entre 1985 y 1991, diferentes legisladores pidieron que la Ley 23109 se hiciese efectiva $y$, una vez que se reglamentara, se pusieran en marcha las distintas soluciones pues el problema en cuestión seguía existiendo. En ese sentido, Britos afirmó que "si bien la política de la miseria que instrumentó el proceso militar ha agravado la situación de todo el pueblo argentino, con su secuela de problemas sociales, no es menos cierto que nuestra sociedad toda está en deuda con los soldados que todo lo brindaron"(15). A la par, Liliana Gurdulich de Correa (Partido Justicialista) puso énfasis en que

...las consecuencias físicas y psíquicas, por las duras condiciones impuestas por el conflicto, comprometen en grado sumo a toda la comunidad y el Estado nacional en una acción efectiva para la recuperación de los excombatientes a los que debemos permanente reconocimiento. ${ }^{(16)}$
Lo cierto es que la reglamentación se llevó a cabo en 1988 a través de un decreto que intentó anticipar el campo de movilidad del veterano que conectaría la convocatoria nacional con su lugar de residencia y sus condiciones de trabajo (vía la gratuidad del transporte y la obligatoriedad de licencias por parte de empleadores), y a la vez con la realización efectiva del reconocimiento médico. Este último, estaría a cargo del Ministerio de Salud y Acción Social ${ }^{(17)}$, no obstante

\begin{abstract}
...hasta tanto no se ejecute la convocatoria nacional obligatoria prevista [...] los veteranos de guerra que padezcan secuelas producidas como consecuencia de las acciones bélicas, podrán presentarse voluntariamente, previa solicitud, a las autoridades militares, a fin de ser sometidos a examen por las Juntas de Reconocimiento Médico de cada Fuerza. ${ }^{(17)}$
\end{abstract}

Asimismo, el diagnóstico de las juntas debían sostenerse en el dictamen de la justicia militar con el fin de "determinar la relación de la afección con el servicio, si las hubiere"(17); a la par, luego de definir "el estado actual, secuelas y tratamiento del paciente" tocaría el turno a la Sanidad Militar (de cada Fuerza) comprobar el diagnóstico de los civiles y llevar a cabo la asistencia que "comprenderá las coberturas médicas, paramédicas y de apoyo de medicamentos en forma totalmente gratuita para el afectado"(17). Finalizado el tratamiento, el veterano debía concurrir nuevamente al reconocimiento médico que convalidaría el alta médica y el grado de recuperación de quienes sean definidos con algún grado de incapacidad. El decreto estableció que "la Junta Médica será la encargada de establecer el tiempo estimado de recuperación del afectado, en el caso de no ser una incapacidad permanente" ${ }^{\prime(17)}$.

La atención médica se efectuará en los siguientes centros por prioridad:

1. Hospitales Militares en su zona de influencia.

2. Hospitales Estatales. 
3. Establecimientos Privados que tengan convenio con las respectivas Obras Sociales.

4. En otros centros de atención.

La trama de diagnósticos, tratamientos y altas, se tejió en idas y vueltas entre el campo civil y el militar, presentándose como el mejor camino posible lo que en realidad era un sitio minado de burocracia, en un momento en el que la salud pública estaba en un proceso continuo de desfinanciamiento, con condiciones similares en la sanidad militar ${ }^{(18)}$. A la vez, la reglamentación aclaró que, una vez determinada la incapacidad del $66 \%$, el veterano conscripto recién adquiría el derecho a incorporarse a la obra social de las Fuerzas o del sistema previsional, con lo cual su cobertura mediante la seguridad social iniciaría una vez que las juntas de reconocimiento médico se hiciesen efectivas. Si bien el decreto generó una interpelación al interior de las Fuerzas Armadas, la convocatoria nacional que debía estar a cargo del Ministerio de Defensa no se efectuó, y los casos de reconocimiento médico que se llevaron a cabo fueron los individuales.

No obstante, el instrumento legal generó modos de pensar el problema de salud en, al menos, dos frentes: la determinación de las secuelas que la guerra generó a través de un diagnóstico convocado por el Estado Nacional y el resarcimiento concomitante; y el hecho de que los veteranos no ostentaban cobertura de salud per se a menos que tuvieran una relación laboral o una pensión por discapacidad, lo que les imposibilitaba el acceso al mercado de trabajo. Aunque el primero generó intervenciones y una latencia que continúa en la actualidad, fue sobre el segundo objeto de pensamiento que se centró la productividad de prácticas posteriores bajo una modalidad de protección focalizada que se patentizó entre junio y septiembre de 1990, bajo la presidencia de Carlos Menem (1989-1999) por el Partido Justicialista.

A fines de junio de ese año, la Administración Nacional de Seguros de Salud (ANSSAL) resolvió "garantizar a todos los ex-combatientes [...], sus grupos familiares primarios y a los grupos de los familiares primarios de los muertos en combate cobertura médicoasistencial"(19) cuando estos no tuvieran obra social. En sus considerandos, el texto motivó la garantía en la situación de los veteranos y su necesidad de amparo, y planteó el objetivo de conectarse con organismos competentes para determinar las características de los beneficiarios. Tres meses después, esa conexión tuvo mayor entidad: la Federación de Veteranos de la Guerra de Malvinas, organismo creado a principios de la década de 1990, con fuerte conexión con los sectores militares vinculados a la guerra y, según Federico Lorenz, muy cercanos al gobierno de Carlos Menem ${ }^{(20)}$. El Ministerio de Salud y Acción Social, el 14 de septiembre, emitió la Resolución 1605/90 que incorporó a los veteranos como población objeto de los programas sociales ${ }^{(21)}$, y que tendría como órgano de aval, informe y asistencia a la Federación de Veteranos de la Guerra de Malvinas. El documento sostuvo su práctica en que los veteranos "si bien comparten la mayoría de las necesidades, derechos y obligaciones del resto de la población, por sus vivencias [...] y especialmente por algunas de sus secuelas físicas y psicológicas, tienen otras necesidades, inéditas para el pueblo Argentino en los últimos cien años", por lo cual es imperativa una atención diferencial. Si bien la resolución no aclaró el tipo y carácter del programa, lo cierto es que actualizó y ensambló como argumento ético a las secuelas y la prioridad con un tipo de política focalizada.

Ese mismo año, el diputado César Jaroslavsky (Unión Cívica Radical) presentó un proyecto de pensión vitalicia que luego se convertiría en la Ley 23848, la primera sobre pensiones de guerra. Por primera vez desde finales del conflicto, una práctica de gobierno se apoyó en datos, pues el diputado sostuvo su intervención en un censo elaborado en 1989 por excombatientes de la provincia de Buenos Aires el cual reflejaba

...que un elevado porcentaje carece de vivienda propia, así como también que la gran mayoría no tiene trabajo estable ni pueden acceder a una asistencia sanitaria digna por no poseer los medios 
económicos o sociales para lograrlo [...] y en muchos casos han optado por el suicidio, el delito como salida individual y la marginación de nuestro sistema de vida". (22)

En otras instancias, el cuerpo de conocimientos para presentar diversas intervenciones y problemas (no solo de salud) partía del sentido común, y no se sostenía en números ni en estudios sobre posibles impactos, por lo cual el texto planteaba una novedad. A su vez, el mismo Jaroslavsky puso énfasis durante el debate en la necesidad de "enfrentar el reclamo de los que quedaron lisiados y de quienes nos vienen a decir que 103 combatientes se suicidaron el mes pasado". De esta forma, el suicidio ingresó como dato, como riesgo extremo $\mathrm{y}$, sobre todo, como número incierto; tríada de funcionamiento que no abandonará a lo largo de los años.

Al año siguiente, dos textos anclados en reglamentaciones diferentes aportaron a la difusa situación sanitaria y de atención de los veteranos. Mediante la Resolución 452/91 de mayo de 1991, el Instituto Nacional de Servicios Sociales para Jubilados y Pensionados (INSSJyP) estableció la afiliación a toda persona que se encontraba tramitando la jubilación o pensiones, exceptuando a los veteranos de guerra, "quienes no pueden afiliarse hasta tanto obtengan la pensión que otorga el Instituto de Ayuda Financiera para el Pago de Retiros y Pensiones Militares o la Gerencia de Protección Social" (Artículo 3, Resolución 452/91), es decir, aquella habilitada por la Ley 23109 y el Decreto 509/88. Paralelamente, y anclado sobre el problema del reconocimiento médico, la Secretaría de Salud resolvió en noviembre de 1991 poner en funcionamiento un plan de activación de las juntas, "dada la finalidad del Servicio Social de crear una actitud crítica respecto a las causales de los problemas sociales, contribuyendo a que los individuos grupos y comunidades organicen las acciones y recursos que apunten a prevenirlas y/o solucionarlas"(23).

Para ello, se elaboraron normas de procedimiento sostenidas también en la Ley 23109, a cargo de la Dirección Nacional de
Delegaciones Sanitarias Federales, donde funcionarían las Juntas de Reconocimiento Médico. En principio, y al igual que las garantías y programas sociales, las listas y avales estarían a cargo de la Federación de Veteranos, que oficiaría de conexión entre el Ministerio de Salud y los veteranos de guerra. A su vez, el documento quitó a los hospitales militares (primeros en importancia según el Decreto 509/88) y se focalizó en hospitales públicos y las delegaciones federales de cualquier jurisdicción. Asimismo, se obvió la convocatoria nacional, optando por solicitudes individuales a interés de cada veterano "acompañando la misma de una historia clínica en la eventualidad de que el mismo se encontrase bajo asistencia médica como consecuencia de la cual reclama los beneficios"(23), y a partir de esa historia clínica se activarían las especialidades médicas que los miembros ad hoc de la junta deberían tener. A la par de los médicos que dictaminarían sobre estado, secuelas y tratamiento, el reconocimiento debía incorporar un profesional de servicios sociales que tendría como función "facilitar el proceso de resocialización del individuo"(23).

Este plan elaborado como un recorte, pero en continuidad del Decreto 509/88, se puso en operaciones varios años después y de manera inconexa. No obstante esto, cabe señalar que la Resolución 130/91 coincidió con dos modificaciones sustanciales en políticas de salud: la transferencia de más de veinte hospitales regulados por el Ministerio de Salud de la Nación (que se sumaron a los 65 descentralizados entre la década de 1970 y 1980), que llevaron a un colapso financiero a las provincias, mayores problemas en la atención, a la par de generar descoordinación entre los niveles federales y provinciales ${ }^{(24)}$, y el inicio de un proceso de reforma que excluiría al sector público y significaría un cambio en la conexión entre salud y protección social(25). Respecto al primero, la focalización en hospitales públicos y delegaciones federales en un momento de descentralización, claramente atentaría contra la concreción del reconocimiento médico. Respecto al segundo, sus contradicciones generarían impactos en otras prácticas de gobierno sobre los veteranos. 
En junio de 1992, el INSSJyP resolvió “otorgar la afiliación provisoria [...] a Veteranos de Guerra, conforme lo preceptuado por la Ley $23848^{\prime \prime 26)}$ siempre que este demuestre su condición certificada por el Ministerio de Defensa, sustituyendo de esta manera la Resolución 452/91 que lo impedía, y conectando al PAMI con la nueva ley de pensiones. Respecto al problema sanitario, este instrumento significó el primer traspaso de coberturas: ya no era necesaria la junta de reconocimiento y la demostración de incapacidad para poder tener protección de salud; la simple certificación de la condición de veteranía habilitaba el trámite. Ese mismo año, el diputado Victorio Bisciotti (Unión Cívica Radical) presentó un cuadro de situación centrado en el estudio realizados en 1989 por el Centro de Ex Soldados Combatientes en Malvinas (CESCEM) de Capital Federal (citado por el diputado Jaroslavsky en 1990), donde se señalaba que

...en materia de salud, las secuelas físi-
cas y psíquicas de la guerra habían pro-
ducido 32 suicidios y 36 detenidos en
diversas cárceles. Aparentemente, las
referidas situaciones no habrían cam-
biado en forma sustantiva desde el
momento en que fuera realizada la pre-
cedentemente aludida encuesta. ${ }^{(27)}$

En 1993, la Federación de Veteranos de la Guerra de Malvinas, auspiciada por el INSSJyP y la Presidencia de la Nación, dio a conocer algunos datos a modo de informe sobre aquella situación, sostenidos en una muestra de 150 veteranos, la cual arrojó que "el $85 \%$ de los veteranos de guerra no ha tenido revisación médica una vez finalizado el conflicto, y el $80 \%$ no ha tenido revisación médica alguna durante estos últimos doce años"(28). Al mismo tiempo, se señaló falta de experiencia en los profesionales médicos, sobre todo en la comprensión de la problemática específica; a su vez, se hizo énfasis en que "la no concurrencia de los veteranos de guerra a los centros de atención de salud, se motiva en la falta de respuestas inmediatas a sus problemáticas"(28), lo cual generó descreimiento de su parte. La problemática no comprendida se enfocó en dos aspectos: la psicológica (con un $76 \%$ de encuestado con esos síntomas) y la osteoarticular (con un $61 \%$ ) que incluyó artralgias (dolor e inflamación en articulaciones) y pie de trinchera, es decir derivas que en el escrito se vincularon la acción en combate y en las trincheras (pozos de zorro).

De esta manera, prioridades, secuelas y situaciones se mostraron en un camino paralelo a la intervención real sobre los veteranos. Simultáneamente, de ese vacío de acción en el reconocimiento médico y la atención emergió como problema la especificidad, atravesada por la duplicidad de rangos de cobertura. El veterano ya no tenía que mostrar sus marcas de guerra y certificarlas; no obstante, estas marcas reingresaron como dato señalando una novedad: la necesidad de una atención focalizada y específica sobre ellas.

\section{EL DERECHO Y LA ESPECIFICIDAD DE ATENCIÓN}

En 1993, año del informe de la Federación de Veteranos de la Guerra de Malvinas, se presentaron al Congreso dos proyectos importantes que conectaron la veteranía y la salud como problema. El primero de ellos, de los diputados José Manuel Corchuelo Blasco y Graciela Camaño (Partido Justicialista), proponía otorgar el "derecho a hacer uso de los servicios que brinda el Instituto Nacional de Servicios Sociales para Jubilados y Pensionados (INSSJP) a toda persona con beneficio acordado conforme al régimen de la Ley 13478 (Pensiones a la Vejez y por Invalidez), la Ley 23746 (Pensión a madres de siete hijos) 23.109 (Beneficios a ex soldados combatientes de Malvinas), y la Ley 23466 (Pensión para menores de 21 años de progenitores desaparecidos), a partir del momento de su reconocimiento(29).

En momentos en los que el organismo que se presentaba como representativo del sector veterano (nos referimos a la Federación de Veteranos) mostró datos sobre la especial 
problemática, paradójicamente coincidió con la ubicación de los veteranos en el mismo rango que otros sujetos en riesgo de desocialización (nos referimos a "colecciones de individuos" a decir de Castel, esto es tipologías utilizadas para referirse no a colectivos sino a segmentos de excluidos indicados como desintegrados o en riesgo de desintegración social, es decir desocializados) ${ }^{(30)}$.

Para obtener la afiliación, el viejo, el inválido, la madre de siete hijos, el hijo de desaparecido o el veterano, todos beneficiarios de pensiones no contributivas (PNC) debían concurrir al Instituto con el comprobante de su condición. Por otra parte, la Federación presionó para que el Ministerio de Salud activase por primera vez la Junta de Reconocimiento Médico, lo cual se realizó parcialmente a mediados de 1993, ya que solo se conformaría la Junta por iniciativas individuales y caso por caso, obviando la convocatoria nacional obligatoria. Al año siguiente, surgieron varios proyectos que recuperaron denuncias públicas (de la propia Federación de Veteranos de la Guerra de Malvinas) respecto a la actuación de las Juntas, como el de Carlos "Chacho" Álvarez (Frente Grande) quien solicitó un informe para corroborar por qué "la mayoría de estos veteranos no fueron sometidos a una revisión médica para poder determinar el grado y la causa de su invalidez"(31). No obstante, las respuestas estatales no se materializaron.

Sobre ese panorama, el interventor del INSSJP, Carlos Alderete, firmó en 1994 el primer programa social destinado exclusivamente a veteranos, sostenido en la red de organizaciones del sector (unas 170 según el documento) puesto que "las particularidades vivenciales, de edad y de experiencia de vida de la población de Veteranos de Guerra de Malvinas no facilita su integración en las entidades conformadas por jubilados y pensionados"(32). El texto creó el Registro Nacional de Entidades de Veteranos de Guerra de Malvinas con el objetivo de canalizar los beneficios como "Programas de Turismo Social, Programas de Mini-turismo, Programas de Recreación y Deporte, Programas de Cultura, Programas de Capacitación y Educación,
Programa de Ayuda Terapéutica para Veteranos de Guerra y su grupo familiar, Plan materno-infantil (guardería)"(32). Al conectar a las organizaciones en el mismo rango que las de jubilados, pero diferenciándolas, la especificidad como problema se actualiza ante una tecnología como la desplegada por el INSSJyP cuyo destinatario tipo era el jubilado. Asimismo, el Ministerio del Interior puso en marcha un plan de salud específico, que llegó a cubrir a unos 2.000 veteranos (sobre una base total de, en ese momento, 12.000) pero se discontinuó al poco tiempo ${ }^{(33)}$.

Pese a estas dos intervenciones, al año siguiente, la diputada Dulce Granados (Partido Justicialista) solicitó la implementación de "un plan de apoyo psicológico específico para los excombatientes de la guerra de las Malvinas [...] para que puedan superar este traumático hecho para sus vidas"(34). El respaldo solicitado por Granados se apoyó en versiones periodísticas que contabilizaron 200 suicidios en 13 años. Como dijimos anteriormente, el suicidio como riesgo extremo de desafiliación y argumento de intervención, empieza a cobrar cada vez mayor relevancia a medida que se pide especificidad en el tratamiento. En sus prácticas, la conexión ascendente entre el número indefinido (que sigue existiendo puesto que no hay cifras oficiales de suicidios, aunque algunas organizaciones elevan el número a más de 500) y sostenido en rumores de suicidios generó un anudamiento en los sueños de los profesionales políticos para pensar modos de intervención, no así para proponer estudios que permitan constituir el problema de forma diferente.

En 1995 el gobierno nacional eliminó la posibilidad de que los beneficiarios del sistema de seguros de salud puedan afiliarse a más de una obra social y las funciones de asistencia social se transfirieron del INSSJyP, mientras que las pensiones no contributivas (que hasta ese momento cubría la vejez, familiares de desaparecidos, madres de siete hijos, inválidos y veteranos) pasaron a la recientemente creada Secretaría de Desarrollo Social, hasta ese momento, a cargo de la Administración Nacional de la Seguridad Social (ANSES). A la par, la cobertura médica de los 
pensionados pasaría a manos de dicha Secretaría, excepto quienes se encontraban bajo la Ley 23848, es decir, los veteranos de guerra (Artículo 19), que continúan en el PAMI ${ }^{(35)}$, a los que se agregarían los pensionados por invalidez. Este acto confuso de ingresos y egresos de protecciones y administraciones, fue la piedra angular de un sistema de cobertura focalizada que a la larga se llamaría Programa Federal (PROFE). Volveremos sobre este punto más adelante.

A raíz de Decreto 292/95, el diputado Guillermo Estévez Boero (Unidad Socialista) solicitó saber qué medidas se tomarían para no discontinuar los servicios médicos y otras prestaciones derivadas del cambio, ya que todos "los beneficiarios son personas de grandes carencias económicas y de salud y por lo tanto más apremiadas en cuanto a su necesidad de recibir una ayuda que en este caso la sociedad en su conjunto"(36). Quitando el hecho de que el cambio de cobertura médica no afectó a los veteranos (cuestión no destacada por el autor del texto), el diputado señaló que a raíz del Decreto se congelaron las tramitaciones de pensión y beneficios previsionales hasta el 1 de enero de 1996. Este punto fue aclarado en la Resolución 69/95, que mantuvo bajo la órbita de ANSES las pensiones y sus solicitudes hasta que se haga efectiva la transferencia a la Secretaría de Desarrollo Social. En medio de estas tensiones, Corchuelo Blasco volvió a presentar su proyecto de ley para brindar -como un derecho- la cobertura de salud a pensionados por vejez, maternidad de siete hijos, menores hijos de desaparecidos y veteranos de guerra ${ }^{(37)}$.

Dos aspectos merecen destacarse sobre este derecho, como gobierno de los no contributivos: primero, puso como problema inicial la posibilidad de una mala práctica administrativa, al señalar que la cobertura inicia una vez concretada la pensión, por lo cual su objetivo era otorgar el beneficio al momento de aceptación de la solicitud; segundo, respecto a los veteranos, al no anclarse en la ley de pensiones de guerra, sino en la de beneficios, que cubría sanitariamente solo a discapacitados, el derecho de uso debía pasar por el camino burocrático de los dictámenes de junta de reconocimiento, cuestión que, como vimos, tuvo serios problemas. En ese sentido, el proyecto, pese a su objetivo inclusivo, actualizaba un conflicto. La presentación fue aprobada sin modificaciones y pasó al Senado, donde se sancionó en noviembre de 1996 bajo la Ley 24734.

En el otro plano del problema, la pregunta sobre qué hacer con la cobertura de salud de los pensionados no contributivos seguía produciendo respuestas. En 1997, el Decreto 197/97 del Poder Ejecutivo dispuso el fin de la intervención del INSSJyP (que databa de 1994) y señaló que la atención de prestaciones médicas para veteranos y beneficiarios por invalidez pasarían desde 1998 a la órbita de la Secretaría de Desarrollo Social pudiendo optar libremente entre el PAMI, la misma Secretaría o las obras sociales correspondientes a su actividad (en caso de que las tuvieren). Meses antes, el jefe de Gabinete, Jorge Rodríguez, anticipó el traslado al afirmar que la inclusión de las pensiones no contributivas no atentó contra la eficiencia del sistema, sino que "complicó la gestión dada la necesidad de tener una variada oferta prestacional"(38), quitando el plano de los derechos y ubicando la cobertura bajo parámetros de mercado. En este sentido, la especial problemática de los veteranos no impidió que puedan abstraerse al reformismo menemista. Sin embargo, la libre elección generó una serie de contradicciones de jurisdicción. En septiembre de 1997, se reglamentó la Ley 24734 (derecho a cobertura) que limitó esa libertad, pues sancionó que la cobertura de la Secretaría de Desarrollo se haría efectiva "siempre que no gozaren o tuvieren derecho a gozar de las prestaciones que brinda el Instituto Nacional de Servicios Sociales para Jubilados y Pensionados o cualquier otra Obra Social" ${ }^{\prime(39)}$. De esta forma, se presentaba como mejor camino posible optar por el PAMI pues el acceso no dependía del dictamen de discapacidad de la junta. Tener el derecho fue el argumento básico de desplazamiento.

Removido por completo el problema del reconocimiento médico en el plano de la cobertura, entre noviembre de 1997 y enero de 1998 el armado tecnológico sobre la atención 
de salud de los veteranos terminó de cobrar forma (aquí consideramos como tecnología a las técnicas y procedimientos que habilitan la cobertura). En principio, se aprobó el formulario para optar por una obra social, en la que parte de los requisitos era ser "titular de uno o varios beneficios previsionales nacionales o titular de una pensión no contributiva otorgada por invalidez o excombatientes de Malvinas" ${ }^{\prime(40)}$. A la par, se aprobaron dos leyes que consolidaron la remoción: la Ley 24901, que reguló la cobertura médica y la atención integral para discapacitados, que incluyó como población beneficiaria a excombatientes y la Ley 24938 de presupuesto para 1998 que, en su artículo 77, transfirió definitivamente la atención de las prestaciones médicas y sociales de las pensiones no contributivas en los casos de invalidez y ex combatientes de Malvinas a la órbita de la Secretaria de Desarrollo Social.

Podríamos afirmar que, si el gobierno (en los términos foucaulteanos de la conducción de conductas) se funda en el problema contradictorio de conducir ciudadanos libres ${ }^{(41)}$, en el plano sanitario vemos un tablero de libertades altamente mediadas. Amén de estos aspectos, el problema del tipo de atención implicado en la cobertura (su especificidad) seguía presente pues, salvo la experiencia fugaz de 1994, no existían programas especiales dirigidos al sector. Como dijimos, el argumento común para establecer la especificidad como problema en cuestión era el riesgo de suicidio. En ese sentido, Juan Ramón Aguirre Lanari (Partido Liberal de Corrientes) solicitó -como lo había hecho Dulce Granados años atrás- un “programa de asistencia integral médica y psicológica para los excombatientes" ${ }^{\prime \prime 2)}$, denunciando que un $90 \%$ no recibió atención médica desde el fin del conflicto, que más de 200 veteranos decidieron quitarse la vida, y dando cuenta de una noticia mediática en la que se menciona una patología específica de la guerra: el estrés postraumático (PTSD, por sus siglas en inglés), por el cual un "fenómeno que ocurría más allá de nuestras fronteras, como por ejemplo, las consecuencias que trajo la guerra de Vietnam en los Estados Unidos de América, hoy se ha instalado entre nosotros" ${ }^{\prime(42)}$.
En ese sentido, el primer plan de tratamiento específico mediante el PAMI inició en noviembre de 1997 cuando el INSSJyP contrató a la empresa Cobertura Federal de Salud SA (COFESA) para brindar atención al sector. Este acuerdo fue puesto en duda a principios de 1998 por las diputadas Elisa Carrió y Miriam Curletti (Unión Cívica Radical), señalando malversación de fondos ${ }^{(43)}$ por parte del directorio y puntualmente su presidente, Víctor Alderete a quien denunciaron penalmente. Ante estas denuncias, ese mismo año, la Federación de Veteranos de Guerra de Malvinas presentó un proyecto replicado por varios legisladores, en el que hicieron hincapié en la necesidad de continuar bajo la órbita de dicha obra social. El primero en recuperar el pedido fue Carlos Snopek (Partido Justicialista) quien citó directamente lo solicitado por la Federación, señalando que el INSSJyP ha "diseñado e implementado un plan de atención de características concernientes a la especificidad de la problemática posbélica y que dicha programación asistencial favoreció la reinserción

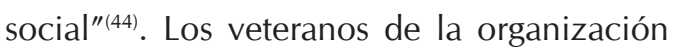
se consideraron satisfechos "después de pasar una década sin atención", por lo cual el diputado solicitó que se asegure la libre elección entre el Programa Federal y el PAMI. Por su parte, Graciela Camaño (Partido Justicialista) presentó el proyecto de ley con el pedido original que no tenía medias tintas, ya que proponía que los veteranos pensionados bajo la Ley 23848, Ley 24652 y Ley 24892 siguieran en la órbita del INSSJyP, "a fin de garantizar la continuidad de los programas médico-asistenciales" ${ }^{\prime(45)}$. En sus fundamentos, se justificó no solo el impacto que tuvo el programa en la reinserción, sino también su ínfima incidencia financiera, pues los veteranos (y sus familias) "comprenden un número de 31.000 afiliados, con posibilidad de incremento en 15.000 más, lo que implica un porcentaje mínimo del padrón total del PAMI que alcanzaría apenas el 0,5\%".

En paralelo, la diputada Dulce Granados (Partido Justicialista) mostró preocupación ante la "posibilidad de que se cancelen las diferentes coberturas médicas asistenciales, 
pensiones, así como también cualquier otro

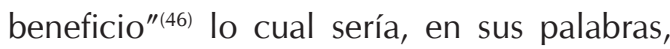
retroceder en las conquistas sociales. A fines de septiembre de 1998, la cabeza de la Jefatura de Gabinete, Jorge Rodríguez, dio coherencia a estas prácticas anunciando un plan nacional especial para veteranos fundado en sus "particulares características de demanda asistencial" $^{\prime(47)}$. El 20 de octubre de 1998, la Cámara de Diputados aprobó el proyecto de Camaño (considerando todos los demás), el cual recién fue tratado en el Senado en junio de 1999, donde se le dio curso con una leve modificación (se quitó la fuente de financiamiento que originalmente era rentas generales) y casi sin discursos, a excepción del senador Omar Vaquir (Partido Justicialista) quien manifestó que

\begin{abstract}
...en un acto de justicia queremos garantizar la cobertura médica del PAMI a veteranos de guerra de Malvinas, de modo que no haya solamente cruces blancas y lisiados, sino que quienes siguen siendo ciudadanos útiles a la Patria tengan el reconocimiento y la asistencia médica que se merecen. ${ }^{(48)}$
\end{abstract}

La ley de continuidad dentro del PAMI (Ley 25210) se promulgó en diciembre de 1999, coincidiendo con el mes de asunción presidencial de Fernando De la Rúa (Unión Cívica Radical), sucesor de Carlos Menem.

En ese sentido, conseguir la especificidad de atención fue una prerrogativa ante el colapso del PAMI y las denuncias de corrupción respecto a la protección de los excombatientes. En junio de 2000, la Jefatura de Gabinete emitió una decisión administrativa desde la cual aseguró financieramente la continuidad de la cobertura del INSSJyP para el sector (y los pensionados por invalidez) ${ }^{(49)}$ y dio nacimiento a la Subgerencia del Veterano de Guerra cuyo fin fue implementar un programa de política de salud específico ${ }^{(50)}$. No obstante, los veteranos afiliados al PAMI tuvieron que esperar hasta 2003 para que el problema de la especialidad de atención cobrara forma de respuesta concreta y específica mediante un programa de gobierno, aunque su tratamiento excede las pretensiones de este escrito.

\section{CONCLUSIONES}

Este trabajo no se propuso desarrollar el éxito o fracaso de las políticas públicas de salud sobre los veteranos de la guerra de Malvinas, sino analizar las formas en que esas políticas presentaron los problemas que venían a resolver. En ese sentido, decíamos en la introducción que nos planteamos describir los modos de problematización política que conectaron la especial problemática de los partícipes de la guerra con la salud como una de las respuestas. En torno a esto, pudimos apreciar que la red de prácticas desplegada en torno a la salud de los veteranos como una necesidad de intervención estatal se centró en dos modos de problematización. Una parte de las prácticas hizo énfasis en la necesidad de una convocatoria general y totalizadora a partir de las juntas de reconocimiento médico; la otra, centró sus esfuerzos en la calidad de cobertura de salud y los grados de dependencia de esta respecto a otras circunstancias (discapacidad, asistencia). Ambas se montaron en una serie de marcas, en principio señaladas como secuelas (físicas y psíquicas) pero que luego cobraron mayor nitidez en el diagnóstico par excellence que identificaría al veterano separándolo del resto de los sujetos sociales: el estrés postraumático y el riesgo del suicidio.

Estos modos no operan en este trabajo como modelos explicativos por fuera de las propias prácticas que le dan forma, y sus conexiones con concepciones e intereses propios del sistema de salud argentino necesitan ser profundizadas. En todo caso, el trabajo se presenta como un diagnóstico de un proceso que dialoga tanto con el desarrollo histórico del propio sistema y con el devenir de una historia específica: la del veterano y su lugar en el pensamiento político argentino en la posguerra.

Identificamos dos momentos que iniciaron con una propuesta de acción estatal a gran escala que permitió imaginar 
diagnósticos en serie mediante las juntas de reconocimiento médico en delegaciones federales, las que posibilitarían reparaciones puntuales a casos individuales (cuyo costo debía ser afrontado por las Fuerzas Armadas, responsables de las secuelas). Esto significa que la cobertura comenzaría una vez establecida la evaluación médica, lo cual dio paso al segundo momento ligado al problema entre la ineficiencia de las juntas y la necesidad de atención personal y familiar de los ex soldados. En ese pasaje pudimos ver la emergencia de la situación especial como problema que, paradójicamente, produjo un derecho especial a la vez que lo ligó a otros sujetos también al borde del abismo social (madres de siete hijos, incapacitados, familiares de desaparecidos, personas sin recursos y en grados avanzados de vejez).

Este proceso coincide con el que se dio partir de la década de 1980, que produjo una crisis del modelo de integración que poseía el país, que dio origen a una etapa de lucha contra la pobreza a través de programas focalizados e híper especializados sostenidos en la tipificación del sujeto vulnerable, al

...privilegiar el acceso a las familias con responsables económicos desempleados, mujeres embarazadas, niños y ancianos [...] además se incluían aspectos de "promoción" asociados al estímulo de la "participación comunitaria" y del ejercicio de la solidaridad. ${ }^{(51)}$

Podríamos afirmar que, en el hiato entre la especificidad y la asistencia, fue produciéndose el camino a una atención especial que tuvo en el PAMI su principal objetivo y, en el corrimiento del diagnóstico previo, su novedad. No obstante, el tratamiento específico tardó más de 20 años en estabilizarse a la par que se diluyeron las posibilidades de que las juntas de reconocimiento realmente se concreten.

La conexión establecida entre salud y veteranía por las diversas prácticas analizadas muestra que lo que se jugó en las definiciones de sus problemas de vida se enmarcó en una suerte de segmentación o focalización poblacional.
Esta focalización, que Álvarez Leguizamón ${ }^{(52)}$ denominó focopolítica, aún se sostenía en metas reintegradoras ligadas a imaginarios que lentamente iban perdiendo espacio en el pensamiento político. Grondona señala que, entre 1983 y 1989, se sucedieron una serie de proyectos y debates parlamentarios sobre la situación social que marcaron una grilla de vulnerables:

...a) los jóvenes que ingresan al mercado de trabajo [...] b) los desempleados con carga de familia [...] c) las mujeres jefas de familia sin empleo [...] d) los desempleados mayores [...] e) las mujeres embarazadas desocupadas [...] f) los minusválidos [...] g) los exdetenidos [...] los migrantes con dificultades de inserción en el mercado de trabajo. ${ }^{(52)}$

A nuestro entender, la situación especial de los veteranos se anticipó en este juego de excepcionalidades, ya que tempranamente se los señaló a partir de su priorización y, coincidentemente, también se los ubicó en el umbral entre una forma de entender la reinserción de manera integral y una de gobierno focalizado.

Es decir, las políticas públicas pensadas y desarrolladas en torno a los veteranos se ligaron fuertemente a las focalizadas, mediante las cuales las prácticas de salud pública se acercan a las asistenciales, y que caracterizan en gran medida los modos neoliberales de pensar los problemas sociales. En ese sentido, lo que se produjo a nivel estatal es un gobierno de las situaciones especiales, sostenido por el planteo de una necesaria reinserción social, en el que el veterano de guerra formó parte de un staff, junto a diversos segmentos poblacionales, que estaban también al borde de la desafiliación social (pensionados por vejez, inválidos y discapacitados, madres de siete hijos, hijos de desaparecidos, y sobre todo suicidas potenciales). En ese sentido, el pensamiento sobre su marginalidad antes de ser un dato fáctico, sostenido científicamente, se erigió en un sentido común que anticipó la gran mayoría de las resoluciones que se plantearon durante el período analizado. Podemos afirmar que, desde los primeros 
meses de democracia (incluso antes), no hubo olvido o abandono del Estado, sino que se configuró un tipo de intervención cuyas condiciones de realización dependieron de un modo concreto de pensar (y producir) el problema. Desde las primeras prácticas, los veteranos de guerra fueron pensados como desanclados de la totalidad y las diversas propuestas de resolución amplificaron esa caracterización, lo cual conllevó a que su especialidad haya anticipado por tanto tiempo la especificidad de atención. Es decir, en el plano de la salud, la necesidad de tratarlos como marginales anticipó por varios años la urgencia de pensar tratamientos específicos para patologías propias de una guerra.

\section{AGRADECIMIENTOS}

Esta investigación contó con el apoyo de la Beca Interna Doctoral que otorga el Consejo Nacional de Investigaciones Científicas y Técnicas (CONICET) para el proyecto "Procesos de construcción identitaria de los 'combatientes continentales' de Malvinas de Chaco y Corrientes", Período 20142019, Resolución D № 4838.

\section{REFERENCIAS BIBLIOGRÁFICAS}

1. Chao D. Gubernamentalidad y pensamiento político: la salud de los veteranos de la guerra de Malvinas como problema legislativo. deSignis. 2017;(26):243-252

2. Guber R. De "chicos" a "veteranos": memorias argentinas de la guerra de Malvinas. Buenos Aires: Antropofagia-IDES; 2004.

3. Rodríguez A. Entre la guerra y la paz: la posguerra de los excombatientes del Apostadero Naval Malvinas: Experiencias, identidades, memorias. [Tesis doctoral]. La Plata: Universidad Nacional de La Plata; 2014.

4. Foucault M. La gubernamentalidad: Estética, ética y hermenéutica. Barcelona: Paidós; 1999.

5. Bacchi C. Problematizations in health policy: questioning how "problems" are constituted in policies. SAGE Open. 2016;6(2):1-16.
6. Bacchi C. Analysing policy: What's the problem represented to be? Sydney: Pearson Australia; 2009.

7. Chao D. Problematizaciones, problemas representados y gubernamentalidad: Una propuesta analítica para el estudio de las políticas públicas y el Estado. De Prácticas y Discursos. 2019;8(11):123152.

8. Latour B. Reensamblar lo social: una introducción a la teoría del actor-red. Buenos Aires: Manantial; 2008.

9. Foucault M. Historia de la sexualidad 2: el uso de los placeres. Madrid: Biblioteca Nueva; 2012.

10. Centrágolo O. Financiamiento fragmentado, cobertura desigual y falta de equidad en el sistema de salud argentino. Revista de Economía Política de Buenos Aires. 2014;13(8):145-183.

11. Honorable Cámara de Senadores de la Nación Argentina. Proyecto de comunicación $N^{\circ}$ S-848-84, Orden del Día Nº 367, 27 sep 1984. Localizado en: Hemeroteca Revistas, Biblioteca del Congreso de la Nación Argentina.

12. Honorable Cámara de Senadores de la Nación Argentina. Proyecto de ley $N^{\circ}$ S-404-84, Orden del Día N³ 367, 27 sep 1984. Localizado en: Hemeroteca Revistas, Biblioteca del Congreso de la Nación Argentina.

13. Honorable Cámara de Senadores de la Nación Argentina. Dictamen de comisiones, Orden del 
Día Nº 367, 27 sep 1984. Localizado en: Hemeroteca Revistas, Biblioteca del Congreso de la Nación Argentina.

14. Honorable Cámara de Senadores de la Nación Argentina. Diario de sesiones, $27^{\circ}$ reunión del 28 sep 1984. Localizado en: Hemeroteca Revistas, Biblioteca del Congreso de la Nación Argentina.

15. Honorable Cámara de Diputados de la Nación Argentina. Proyecto de declaración $\mathrm{N}^{\circ} \mathrm{S}-62-85$, Diario de Asuntos Entrados $N^{\circ} 13,7$ mar 1985. Localizado en: Hemeroteca Revistas, Biblioteca del Congreso de la Nación Argentina.

16. Honorable Cámara de Senadores de la Nación Argentina. Proyecto de comunicación $\mathrm{N}^{\circ}$ S-115-85, Diario de Asuntos Entrados $N^{\circ} 26,12$ abr 1985. Localizado en: Hemeroteca Revistas, Biblioteca del Congreso de la Nación Argentina.

17. Argentina. Beneficios a Ex Combatientes, Decreto 509/88. Boletín Oficial de la República Argentina.1988;96(26.382):3-4.

18. Frederic S. Las trampas del pasado: las Fuerzas Armadas y su integración al Estado democrático en Argentina. Buenos Aires: Fondo de Cultura Económica; 2013.

19. Argentina, Administración Nacional de Seguro de Salud. Resolución 66/90. Boletín Oficial de la República Argentina. 1990;98(26.913):5.

20. Lorenz F. Las guerras por Malvinas. 2a ed. Buenos Aires: Edhasa; 2012.

21. Argentina, Ministerio de Salud y Acción Social de la Nación. Resolución Ministerial 1605/90 [Internet]. 14 sep 1990 [citado 26 feb 2018]. Disponible en: https://tinyurl.com/yxdyerxe.

22. Honorable Cámara de Diputados de la Nación Argentina. Proyecto de Ley 3187-D-90, Trámite Parlamentario $\mathrm{N}^{\circ}$ 99, 13 sep 1990. Localizado en: Hemeroteca Revistas, Biblioteca del Congreso de la Nación Argentina.

23. Argentina, Secretaría de Salud, Ministerio de Salud y Acción Social de la Nación Argentina. Resolución 130/91 [Internet]. 15 nov 1991 [citado 2 mar 2018]. Disponible en: https://tinyurl.com/yxphfrvz.

24. Centrágolo O. Financiamiento fragmentado, cobertura desigual y falta de equidad en el sistema de salud argentino. Revista de Economía Política de Buenos Aires. 2014;13(8):145-183.

25. Grassi E. Políticas y problemas sociales en la sociedad neoliberal: La otra década infame. Buenos Aires: Espacio Editorial; 2003.
26. Argentina, Instituto Nacional de Servicios Sociales para Jubilados y Pensionados. Resolución 692/91 [Internet]. 24 jun 1992 [citado 2 marzo 2018]. Disponible en: https://tinyurl.com/y2pbukdx.

27. Honorable Cámara de Diputados de la Nación Argentina. Proyecto de resolución 3585-D-92, Trámite Parlamentario $N^{\circ} 113,6$ oct 1992. Localizado en: Hemeroteca Revistas, Biblioteca del Congreso de la Nación Argentina.

28. Federación de Veteranos de Guerra de la República Argentina. Veteranos de guerra y sociedad. Pre-Jornadas Nacionales Veteranos de guerra y sociedad. Ciudad Autónoma de Buenos Aires: Informe FVGM; 1993.

29. Honorable Cámara de Diputados de la Nación Argentina. Proyecto de ley 1268-D-93, Trámite Parlamentario $N^{\circ}$ 33, 15 jun 1993. Localizado en: Hemeroteca Revistas, Biblioteca del Congreso de la Nación Argentina.

30. Castel R. La inseguridad social: ¿Qué es estar protegido? Buenos Aires: Mantial; 2004.

31. Honorable Cámara de Diputados de la Nación Argentina. Proyecto de resolución 4699-D-93, Trámite Parlamentario No 201, 17 feb 1994. Localizado en: Hemeroteca Revistas, Biblioteca del Congreso de la Nación Argentina.

32. Argentina, Instituto Nacional de Servicios Sociales para Jubilados y Pensionados. Resolución 1336/94 [Internet]. 4 nov 1994 [citado 23 mar 2018]. Disponible en: https://tinyurl.com/y22oacor.

33. Silva MA. Salud de los Veteranos de Guerra de Malvinas: miradas, sentires y propuestas. VIII Ciclo Anual de Ateneo Patología Comparada. Santa Fe: Museo Provincial de Ciencias Naturales "F. Ameghino"; 2006.

34. Honorable Cámara de Diputados de la Nación Argentina. Proyecto de declaración 2665-D-95, Trámite Parlamentario $N^{\circ} 85,4$ jul 1995. Localizado en: Hemeroteca Revistas, Biblioteca del Congreso de la Nación Argentina.

35. Argentina, Poder Ejecutivo. Seguridad Social, Decreto 292/95. Boletín Oficial de la República Argentina. 1995;103(28.209):3-6.

36. Honorable Cámara de Diputados de la Nación Argentina. Proyecto de resolución N 4683-D-95, Trámite Parlamentario $\mathrm{N}^{\circ}$ 166, 26 oct de 1995. Localizado en: Hemeroteca Revistas, Biblioteca del Congreso de la Nación Argentina.

37. Honorable Cámara de Diputados de la Nación Argentina. Proyecto de ley Nº 3609-D-95, Trámite 
Parlamentario $N^{\circ}$ 122, 25 ago 1995. Localizado en: Hemeroteca Revistas, Biblioteca del Congreso de la Nación Argentina.

38. Argentina, Jefatura de Gabinete de Ministros de la Nación. Informe del Jefe de Gabinete de Ministros ante el Honorable Congreso de la Nación, Ing. Jorge Rodriguez, Informe $N^{\circ} 11$ [Internet]. 27 sep 1996 [citado 3 mar 2018]. Disponible en: https://tinyurl.com/y3ofujzo.

39. Argentina, Poder Ejecutivo. Servicios de Cobertura Médica, Decreto 945/97. Boletín Oficial de la República Argentina. 1997;105(28.734):1-2.

40. Argentina, Superintendencia de Administradoras de Fondos de Jubilaciones y Pensiones. Resolución 572/97. Boletín Oficial de la República Argentina. 1997;105(28.783):43-61.

41. Gordon C. Racionalidad gubernamental: una introducción. Nuevo Itinerario Revista Digital de Filosofía. 2015;10(X):1-58.

42. Honorable Cámara de Senadores de la Nación Argentina. Proyecto de comunicación Nº S-364-97, Diario de Asuntos Entrados N 24, 7 abr 1997. Localizado en: Hemeroteca Revistas, Biblioteca del Congreso de la Nación Argentina.

43. Honorable Cámara de Diputados de la Nación Argentina. Proyecto de resolución N 6823-D-97, Trámite Parlamentario $N^{\circ}$ 232, 24 feb 1997. Localizado en: Hemeroteca Revistas, Biblioteca del Congreso de la Nación Argentina.

44. Honorable Cámara de Diputados de la Nación Argentina, Proyecto de resolución N 4440-D-98. Trámite Parlamentario N 90, 14 jul 1998. Localizado en: Hemeroteca Revistas, Biblioteca del Congreso de la Nación Argentina.
45. Honorable Cámara de Diputados de la Nación Argentina. Proyecto de ley N 4810-D-98, Trámite Parlamentario $\mathrm{N}^{\circ}$ 106, 5 ago 1998. Localizado en: Hemeroteca Revistas, Biblioteca del Congreso de la Nación Argentina.

46. Honorable Cámara de Diputados de la Nación Argentina, Proyecto de declaración N 5356-D-98. Trámite Parlamentario $N^{\circ}$ 121, 27 ago 1998. Localizado en: Hemeroteca Revistas, Biblioteca del Congreso de la Nación Argentina.

47. Argentina, Jefatura de Gabinete de Ministros de la Nación. Informe del Jefe de Gabinete de Ministros ante el Honorable Congreso de la Nación, Ing. Jorge Rodriguez, Informe N²9 [Internet]. 30 sep 1998 [citado 3 mar 2018]. Disponible en: https://tinyurl.com/y4cew2kh.

48. Honorable Cámara de Senadores de la Nación Argentina. Diario de sesiones, 25 sesión ordinaria [Internet]. 16 jun 1999 [citado 26 feb 2019]. Disponible en: https://tinyurl.com/y586ay7w.

49. Argentina, Jefatura de Gabinete de Ministros. Decisión Administrativa 78/2000. Boletín Oficial de la República Argentina. 2000;108(29.432):2.

50. Silva MA. Salud de los Veteranos de Guerra de Malvinas: miradas, sentires y propuestas. VIII Ciclo Anual de Ateneo Patología Comparada. Santa Fe: Museo provincial de Ciencias Naturales "F. Ameghino"; 2006.

51. Grondona A. 'Tradición' y 'traducción': un estudio de las formas contemporáneas del gobierno de las poblaciones desempleadas en la Argentina. Buenos Aires: Centro Cultural de la Cooperación Floreal Gorini; 2012.

52. Álvarez Leguizamón S. Pobreza y Desarrollo en América Latina. Salta: UNSa; 2008.

\section{FORMA DE CITAR}

Chao D. Especial y marginal: hacia una problematización de la intervención estatal en la salud de los veteranos de Malvinas (1984-2000). Salud Colectiva. 2019;15:e2205. doi: 10.18294/sc.2019.2205.

Recibido: 27 de febrero de 2019 | Versión final: 3 de julio de 2019 | Aprobado: 10 de septiembre de 2019

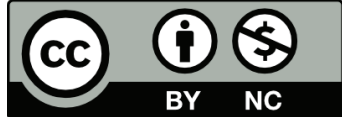

Esta obra está bajo una licencia de Creative Commons Reconocimiento-NoComercial 4.0 Internacional. Reconocimiento - Permite copiar, distribuir y comunicar públicamente la obra. A cambio, se debe reconocer y citar al autor original. No Comercial - Esta obra no puede ser utilizada con finalidades comerciales, a menos que se obtenga el permiso. 